ALEA, Lat. Am. J. Probab. Math. Stat. 13, 711-724 (2016)

DOI: 10.30757/ALEA.v13-28

\title{
Extremes of the supercritical Gaussian Free Field
}

\section{Alberto Chiarini, Alessandra Cipriani and Rajat Subhra Hazra}

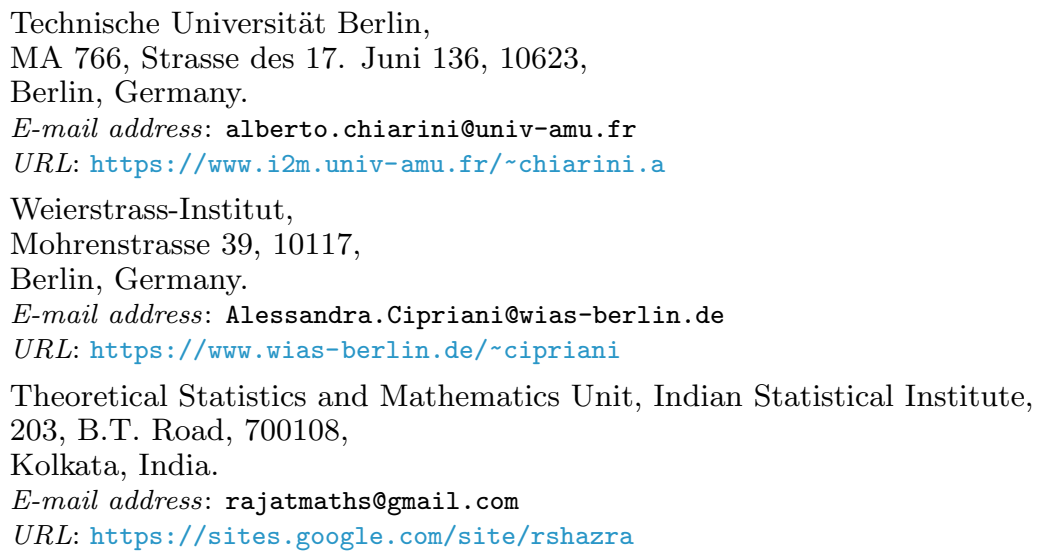

Theoretical Statistics and Mathematics Unit, Indian Statistical Institute, 203, B.T. Road, 700108,

Kolkata, India.

E-mail address: rajatmaths@gmail.com

$U R L:$ https://sites.google.com/site/rshazra

\begin{abstract}
We show that the rescaled maximum of the discrete Gaussian Free Field (DGFF) in dimension larger or equal to 3 is in the maximal domain of attraction of the Gumbel distribution. The result holds both for the infinite-volume field as well as the field with zero boundary conditions. We show that these results follow from an interesting application of the Stein-Chen method from Arratia et al. (1989).
\end{abstract}

\section{Introduction}

In this article we consider the problem of determining the scaling limit of the maximum of the discrete Gaussian free field (DGFF) on $\mathbb{Z}^{d}, d \geq 3$. Recently, for $d=2$, Bramson et al. (2016) showed that the maximum of the DGFF on box of size $N$ converges to a non-trivial limit law, after appropriate recentering. In this case, due to the presence of the logarithmic growth of covariances, the problem is connected to extremes of various other models, for example the Branching Brownian motion and the Branching random walk. In $d \geq 3$, the presence of covariances decaying polynomially changes the setting but the behavior of maxima is still hard to determine Chatterjee (2014, Section 9.6). This dependence also becomes a hurdle

Received by the editors June 9, 2016; accepted July 13, 2016.

2010 Mathematics Subject Classification. 60K35, 60G15, 60G70.

Key words and phrases. Extreme value theory, Gaussian free field, Stein-Chen method.

The first author's research was supported by RTG 1845 . 
in various properties of level set percolation of the DGFF which were exhibited in a series of interesting works (Rodriguez and Sznitman, 2013; Sznitman, 2012; Drewitz and Rodriguez, 2015). The behavior of local extremes in the critical dimension has also been unfolded recently in the papers Biskup and Louidor $(2016,2014)$.

We consider the lattice $\mathbb{Z}^{d}, d \geq 3$ and take the infinite-volume Gaussian free field $\left(\varphi_{\alpha}\right)_{\alpha \in \mathbb{Z}^{d}}$ with law $\mathrm{P}$ on $\mathbb{R}^{\mathbb{Z}^{d}}$. The covariance structure of the field is given by the Green's function $g$ of the standard random walk, namely $\mathrm{E}\left[\varphi_{\alpha} \varphi_{\beta}\right]=g(\alpha-\beta)$, for $\alpha, \beta \in \mathbb{Z}^{d}$. For more details of the model we refer to Section 2. It is well- known (see for instance Lawler, 1991) that for $\alpha \neq \beta, g(\alpha-\beta)$ decays likes $\|\alpha-\beta\|^{2-d}$ and hence for $\|\alpha-\beta\| \rightarrow+\infty$, the covariance goes to zero. However this is not enough to conclude that the scaling is the same of an independent ensemble. To give an example where this is not the case, when $V_{N}$ is the box of volume $N, \sum_{\alpha \in V_{N}} \varphi_{\alpha}$ is of order $N^{1 / 2+1 / d}$, unlike the i. i. d. setting (see for example Funaki, 2005, Section $3.4)$.

The expected maxima over a box of volume $N$ behaves like $\sqrt{2 g(0) \log N}$ as $N \rightarrow+\infty$. An independent proof of this fact is provided in Proposition 2.2 below; this confirms the idea that the extremes of the field resemble that of independent $\mathcal{N}(0, g(0))$ random variables. In this article we show that the similarity is even deeper, since the fluctuations of the maximum after recentering and scaling converge to a Gumbel distribution. Note that in $d=2$ the limit is also Gumbel, but with a random shift (see Bramson et al., 2016, Theorem 2.5, Biskup and Louidor, 2016). The main results of this article is the following.

Theorem 1.1. Let $A_{N}$ be a sequence of subsets of $\mathbb{Z}^{d}$ such that $\left|A_{N}\right|=N$. We define two sequences as follows:

$$
b_{N}=\sqrt{g(0)}\left[\sqrt{2 \log N}-\frac{\log \log N+\log (4 \pi)}{2 \sqrt{2 \log N}}\right] \quad \text { and } \quad a_{N}=g(0)\left(b_{N}\right)^{-1}
$$

so that for all $z \in \mathbb{R}$

$$
\lim _{N \rightarrow+\infty} \mathrm{P}\left(\frac{\max _{\alpha \in A_{N}} \varphi_{\alpha}-b_{N}}{a_{N}}<z\right)=\exp \left(-\mathrm{e}^{-z}\right) .
$$

Note that scaling and centering are exactly the same as in the i. i. d. $\mathcal{N}(0, g(0))$ case, see for example Hall (1982). As in $d=2$, the argument depends on a comparison lemma. We show that in fact the proof is an interesting application of a Stein-Chen approximation result. Not only does the result depend on the correlation decay, but also crucially on the Markov property of the Gaussian free field. We use Theorem 1 of the paper by Arratia et al. (1989) which approximates an appropriate dependent Binomial process with a Poisson process, and gives some calculable error terms. In general showing that the error terms go to zero is a non-trivial task. In the DGFF case, thanks to estimates on the Green's function and the Markov property, the error terms are negligible.

The techinques used for the infinite-volume DGFF allow us to draw conclusions also for the field with boundary conditions. For $n>0$ let $N:=n^{d}$; we consider the discrete hypercube $V_{N}:=[0, n-1]^{d} \cap \mathbb{Z}^{d}$. We define therein a mean zero Gaussian field $\left(\psi_{\alpha}\right)_{\alpha \in \mathbb{Z}^{d}}$ whose covariance matrix $\left(g_{N}(\alpha, \beta)\right)_{\alpha, \beta \in V_{N}}$ is the Green's function of the discrete Laplacian with Dirichlet boundary conditions outside $V_{N}$ (again for a more precise definition see Section 2). The convergence result is the following: 
Theorem 1.2. Let $V_{N}$ be as above and $\left(\psi_{\alpha}\right)_{\alpha \in \mathbb{Z}^{d}}$ be a DGFF with zero boundary conditions outside $V_{N}$ with law $\widetilde{\mathrm{P}}_{V_{N}}$. Let the centering and scaling be as in (1.1). Then for all $z \in \mathbb{R}$

$$
\lim _{N \rightarrow+\infty} \widetilde{\mathrm{P}}_{V_{N}}\left(\frac{\max _{\alpha \in V_{N}} \psi_{\alpha}-b_{N}}{a_{N}}<z\right)=\exp \left(-\mathrm{e}^{-z}\right)
$$

The core of the proof is an application of Slepian's Lemma and also the use Theorem 1.1 with the help of the Markov property. The structure of the article is as follows. In Section 2 we recall the main facts on the DGFF that will be used in Section 3 to prove the main theorem.

\section{Preliminaries on the DGFF}

Let $d \geq 3$ and denote with $\|\cdot\|$ the $\ell_{\infty}$-norm on the lattice. Let $\psi=\left(\psi_{\alpha}\right)_{\alpha \in \mathbb{Z}^{d}}$ be a discrete Gaussian Free Field with zero boundary conditions outside a finite set $\Lambda \Subset \mathbb{Z}^{d}$. On the space $\Omega:=\mathbb{R}^{\mathbb{Z}^{d}}$ endowed with its product topology, its law $\widetilde{\mathrm{P}}_{\Lambda}$ can be explicitly written as

$$
\widetilde{\mathrm{P}}_{\Lambda}(\mathrm{d} \psi)=\frac{1}{Z_{\Lambda}} \exp \left(-\frac{1}{4 d} \sum_{\alpha, \beta \in \mathbb{Z}^{d}:\|\alpha-\beta\|=1}\left(\psi_{\alpha}-\psi_{\beta}\right)^{2}\right) \prod_{\alpha \in \Lambda} \mathrm{d} \psi_{\alpha} \prod_{\alpha \in \mathbb{Z}^{d} \backslash \Lambda} \delta_{0}\left(\mathrm{~d} \psi_{\alpha}\right) .
$$

In other words $\psi_{\alpha}=0 \widetilde{\mathrm{P}}_{\Lambda}$-a. s. if $\alpha \in \mathbb{Z}^{d} \backslash \Lambda$, and $\left(\psi_{\alpha}\right)_{\alpha \in \Lambda}$ is a multivariate

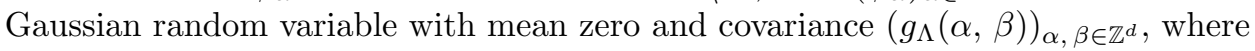
$g_{\Lambda}$ is the Green's function of the discrete Laplacian problem with Dirichlet boundary conditions outside $\Lambda$. For a thorough review on the model the reader can refer for example to Sznitman (2012). One can define also an infinite volume version of this model, namely by $\varphi=\left(\varphi_{\alpha}\right)_{\alpha \in \mathbb{Z}^{d}}$, a centered Gaussian field with covariance matrix $g(\alpha, \beta)_{\alpha, \beta \in \mathbb{Z}^{d}}$ which admits the following random walk representation: if $\mathbb{P}_{\alpha}$ denotes the law of a simple random walk $S$ started at $\alpha \in \mathbb{Z}^{d}$, then

$$
g(\alpha, \beta)=\mathbb{E}_{\alpha}\left[\sum_{n \geq 0} \mathbb{1}_{\left\{S_{n}=\beta\right\}}\right]
$$

Since $g(0)<+\infty$ for $d \geq 3$, it is known Georgii (1988, Chapter 13) that $\varphi$ is the limit as $\Lambda \uparrow \mathbb{Z}^{d}$ of the finite-volume measure $\psi$ in the weak topology of probability measures (for $d \geq 3$ ). With a slight abuse of notation we write $g(\alpha-\beta)$ for $g(\alpha, \beta)$ and also $g_{\Lambda}(\alpha)=g_{\Lambda}(\alpha, \alpha)$.

A key fact for the Gaussian Free Field is its spatial Markov property, which will be used in the paper. The proof of the following Lemma can be found in Rodriguez and Sznitman (2013, Lemma 1.2).

Lemma 2.1 (Markov property of the Gaussian Free Field). Let $\emptyset \neq K \Subset \mathbb{Z}^{d}$, $U:=\mathbb{Z}^{d} \backslash K$ and define $\left(\widetilde{\varphi}_{\alpha}\right)_{\alpha \in \mathbb{Z}^{d}}$ by

$$
\varphi_{\alpha}=\tilde{\varphi}_{\alpha}+\mu_{\alpha}, \quad \alpha \in \mathbb{Z}^{d}
$$

where $\mu_{\alpha}$ is the $\sigma\left(\varphi_{\beta}, \beta \in K\right)$-measurable map defined as

$$
\mu_{\alpha}=\sum_{\beta \in K} \mathbb{P}_{\alpha}\left(H_{K}<+\infty, S_{H_{K}}=\beta\right) \varphi_{\beta}, \quad \alpha \in \mathbb{Z}^{d}
$$


Here $H_{K}:=\inf \left\{n \geq 0: S_{n} \in K\right\}$. Then, under $\mathrm{P},\left(\widetilde{\varphi}_{\alpha}\right)_{\alpha \in \mathbb{Z}^{d}}$ is independent of $\sigma\left(\varphi_{\beta}, \beta \in K\right)$ and distributed as $\left(\psi_{\alpha}\right)_{\alpha \in \mathbb{Z}^{d}}$ under $\widetilde{\mathrm{P}}_{U}$.

As an immediate consequence of the Lemma (see Rodriguez and Sznitman, 2013, Remark 1.3)

$$
\mathrm{P}\left(\left(\varphi_{\alpha}\right)_{\alpha \in \mathbb{Z}^{d}} \in \cdot \mid \sigma\left(\varphi_{\beta}, \beta \in K\right)\right)=\widetilde{\mathrm{P}}_{U}\left(\left(\psi_{\alpha}+\mu_{\alpha}\right)_{\alpha \in \mathbb{Z}^{d}} \in \cdot\right) \quad \mathrm{P}-a . s .
$$

where $\mu_{\alpha}$ is given in (2.1), $\widetilde{\mathrm{P}}_{U}$ does not act on $\left(\mu_{\alpha}\right)_{\alpha \in \mathbb{Z}^{d}}$ and $\left(\psi_{\alpha}\right)_{\alpha \in \mathbb{Z}^{d}}$ has the law $\widetilde{\mathrm{P}}_{U}$.

2.0.1. Law of large numbers of the recentered maximum. Although this can be obtained directly by Theorem 1.1, we think it is interesting to insert an independent proof of the behavior of the maximum of the DGFF.

Proposition 2.2 (LLN for the maximum). Let $V_{N}:=[0, n-1]^{d} \cap \mathbb{Z}^{d}, N:=n^{d}>0$. The following limit holds:

$$
\lim _{N \rightarrow+\infty} \frac{\mathrm{E}\left[\max _{\alpha \in V_{N}} \varphi_{\alpha}\right]}{\sqrt{2 \log N}}=\sqrt{g(0)}
$$

Proof: Using Slepian's lemma and by comparison with independent $\mathcal{N}(0, g(0))$ random variables, the upper bound is immediate. As for the lower bound, we will use Sudakov-Fernique inequality, see Adler and Taylor (2007, Theorem 2.2.3). We first need a lower bound for $\mathrm{E}\left[\left(\varphi_{\alpha}-\varphi_{\beta}\right)^{2}\right]$ : we will apply here the bound

$$
g(\alpha) \leq\left(\frac{c \sqrt{d}}{\|\alpha\|}\right)^{d-2}, \quad\|\alpha\| \geq d
$$

whose proof is provided in Sznitman (2011). The key to obtain the result is to use a diluted version of the DGFF as follows. Consider $V_{N}^{(k)}:=V_{N} \cap k \mathbb{Z}^{d}$, where $k:=\lfloor\log n\rfloor \in\{1,2, \ldots\}$. Note the fact that the expected maximum on $V_{N}$ is lower bounded by that on the diluted lattice $V_{N}^{(k)}$. Now for $\alpha, \beta \in T:=V_{N}^{(k)}$ and $k>d$

$$
\begin{aligned}
& \mathrm{E}\left[\left(\varphi_{\alpha}-\varphi_{\beta}\right)^{2}\right]=2 g(0)-2 g(\alpha-\beta) \stackrel{(2.2)}{\geq} 2\left(g(0)-\left(\frac{c \sqrt{d}}{\|\alpha-\beta\|}\right)^{d-2}\right) \\
& \geq 2\left(g(0)-\left(\frac{c \sqrt{d}}{\lfloor\log n\rfloor}\right)^{d-2}\right)=: 2 \nu(n, d)>0
\end{aligned}
$$

for $n$ large enough. Notice also that $\lim _{\rightarrow+\infty} \nu(n, d)=\sqrt{g(0)}$. Now take $\left(G_{\alpha}\right)_{\alpha \in T}$ to be centered independent Gaussian random variables with variance $\nu(n, d)$. Then from above we get that

$$
\mathrm{E}\left[\left(\varphi_{\alpha}-\varphi_{\beta}\right)^{2}\right] \geq \mathrm{E}\left[\left(G_{\alpha}-G_{\beta}\right)^{2}\right] .
$$

By an application of the Sudakov-Fernique inequality we have, $E\left[\max _{\alpha \in T} \varphi_{\alpha}\right] \geq$ $\mathrm{E}\left[\max _{\alpha \in T} G_{\alpha}\right]$ and hence,

$$
\frac{\mathrm{E}\left[\max _{\alpha \in V_{N}} \varphi_{\alpha}\right]}{\sqrt{\log N}} \geq \nu(n, d) \sqrt{\frac{\log |T|}{\log N}} .
$$


We obtain $\log |T|=d \log \left\lfloor\frac{n}{k}\right\rfloor(1+\mathrm{o}(1))=d \log \left\lfloor\frac{n}{\lfloor\log n\rfloor}\right\rfloor(1+\mathrm{o}(1))$. It follows that $\frac{\log |T|}{\log N}=1+\mathrm{o}(1)$ and

$$
\lim _{N \rightarrow+\infty} \frac{\mathrm{E}\left[\max _{\alpha \in V_{N}} \varphi_{\alpha}\right]}{\sqrt{\log N}} \geq \sqrt{2 g(0)}
$$

\section{Proof of the main result}

The proof of the main result is an application of the Stein-Chen method. To keep the article self contained we recall the result from Arratia et al. (1989).

3.1. Poisson approximation for extremes of random variables. The main tool we will use relies on a two-moment condition to determine the convergence of the number of exceedances for a sequence of random variables. Let $\left(X_{\alpha}\right)_{\alpha \in A}$ be a sequence of (possibly dependent) Bernoulli random variables of parameter $p_{\alpha}$. Let $W:=\sum_{\alpha \in A} X_{\alpha}$ and $\lambda:=\mathrm{E}[W]$. Now for each $\alpha$ we assume the existence of a subset $B_{\alpha} \subseteq A$ which we consider a "neighborhood" of dependence for the variable $X_{\alpha}$, such that $X_{\alpha}$ is nearly independent from $X_{\beta}$ if $\beta \in A \backslash B_{\alpha}$. Set

$$
\begin{gathered}
b_{1}:=\sum_{\alpha \in A} \sum_{\beta \in B_{\alpha}} p_{\alpha} p_{\beta}, \\
b_{2}:=\sum_{\alpha \in A} \sum_{\alpha \neq \beta \in B_{\alpha}} \mathrm{E}\left[X_{\alpha} X_{\beta}\right], \\
b_{3}:=\sum_{\alpha \in A} \mathrm{E}\left[\left|\mathrm{E}\left[X_{\alpha}-p_{\alpha} \mid \mathcal{H}_{\alpha}\right]\right|\right]
\end{gathered}
$$

where

$$
\mathcal{H}_{\alpha}:=\sigma\left(X_{\beta}: \beta \in A \backslash B_{\alpha}\right) .
$$

Theorem 3.1 (Theorem 1, Arratia et al., 1989). Let $Z$ be a Poisson random variable with $\mathrm{E}[Z]=\lambda$ and let $d_{T V}$ denote the total variation distance between probability measures. Then

$$
d_{T V}(\mathcal{L}(W), \mathcal{L}(Z)) \leq 2\left(b_{1}+b_{2}+b_{3}\right)
$$

and

$$
\left|P(W=0)-\mathrm{e}^{-\lambda}\right|<\min \left\{1, \lambda^{-1}\right\}\left(b_{1}+b_{2}+b_{3}\right) .
$$

Let now $A \Subset \mathbb{Z}^{d}$ with $N:=|A|, u_{N}(z):=a_{N} z+b_{N}$, and define for all $\alpha \in A$

$$
X_{\alpha}=\mathbb{1}_{\left\{\varphi_{\alpha}>u_{N}(z)\right\}} \sim B e\left(p_{\alpha}\right) .
$$

A standard tool to determine the asymptotic of $p_{\alpha}$ is Mills ratio:

$$
\left(1-\frac{1}{t^{2}}\right) \frac{\mathrm{e}^{-t^{2} / 2}}{\sqrt{2 \pi} t} \leq \mathrm{P}(\mathcal{N}(0,1)>t) \leq \frac{\mathrm{e}^{-t^{2} / 2}}{\sqrt{2 \pi} t}, \quad t>0 .
$$

This yields $p_{\alpha} \sim N^{-1} \exp (-z)$ as $N \rightarrow \infty$. Since $p_{\alpha}$ is independent of $\alpha$, we suppress the subscript $\alpha$ throughout. We will also use later a bivariate form of 
this bounds in the form proved by Savage (1962, formula (I)): for $\mathbf{X}=\left(X_{1}, X_{2}\right) \sim$ $\mathcal{N}(\mathbf{0}, \Sigma), \mathbf{a}=\left(a_{1}, a_{2}\right) \in \mathbb{R}^{2}$ and $f_{\mathbf{X}}$ the density of $\mathbf{X}$, one has

$$
\mathrm{P}\left(X_{1}>a, X_{2}>a_{2}\right) \leq f_{\mathbf{X}}(\mathbf{a})\left(\prod_{i=1}^{2} \Delta_{i}\right)^{-1}
$$

with $\Delta_{i}:=\sum_{j=1}^{2} a_{i}\left(\Sigma^{-1}\right)_{j i}$. We furthermore introduce $W:=\sum_{\alpha \in A} X_{\alpha}$ and see that $\mathrm{E}[W] \sim \mathrm{e}^{-z}$. Of course $W$ is closely related to the maximum since $\left\{\max _{\alpha \in A} \varphi_{\alpha} \leq u_{N}(z)\right\}=\{W=0\}$. We will now fix $z \in \mathbb{R}$ and $\lambda:=\mathrm{e}^{-z}$. We are now ready to prove our main result.

Proof: Our main idea is to apply Theorem 3.1. The proof will first show that the limit is Gumbel, and in the second part we will prove uniform convergence. To this scope we define, for a fixed but small $\epsilon>0$,

$$
B_{\alpha}:=B\left(\alpha,(\log N)^{2+2 \epsilon}\right) \cap A
$$

where $B(\alpha, L)$ denotes the ball of center $\alpha$ of radius $L$ in the $\ell_{\infty}$-distance. We draw below examples of such neighborhoods when

$$
\alpha \in \partial A:=\left\{\gamma \in A: \exists \beta \in \mathbb{Z}^{d} \backslash A,\|\beta-\gamma\|=1\right\}
$$

and $\alpha \in \operatorname{int}(A)=A \backslash \partial A$.

Convergence. The method is based on the estimate of three terms (cf. Subsec. 3.1).

(i) Recall $b_{1}=\sum_{\alpha \in A} \sum_{\beta \in B_{\alpha}} p^{2}$. Using Mills ratio we have

$$
\begin{aligned}
b_{1} & \leq c N(\log N)^{d(2+2 \epsilon)}\left(\frac{\sqrt{g(0)} \mathrm{e}^{-\frac{1}{2 g(0)} u_{N}(z)^{2}}}{\sqrt{2 \pi} u_{N}(z)}\right)^{2} \\
& =N^{-1}(\log N)^{d(2+2 \epsilon)} \mathrm{e}^{-2 z+\mathrm{o}(1)}=\mathrm{o}(1) .
\end{aligned}
$$

(ii) Recall $b_{2}=\sum_{\alpha \in A} \sum_{\alpha \neq \beta \in B_{\alpha}} \mathrm{E}\left[X_{\alpha} X_{\beta}\right]$. First we need to estimate the joint probability

$$
\mathrm{P}\left(\varphi_{\alpha}>u_{N}(z), \varphi_{\beta}>u_{N}(z)\right) .
$$

Denote the covariance matrix

$$
\Sigma_{2}=\left[\begin{array}{cc}
g(0) & g(\alpha-\beta) \\
g(\alpha-\beta) & g(0)
\end{array}\right]
$$

Note that, for $w \in \mathbb{R}^{2}$, one has

$$
w^{t} \Sigma_{2}^{-1} w=\frac{1}{g(0)^{2}-g(\alpha-\beta)^{2}}\left(g(0)\left(w_{1}^{2}+w_{2}^{2}\right)-2 g(\alpha-\beta) w_{1} w_{2}\right) .
$$

Using $1:=(1,1)^{t}$ we denote by

$$
\Delta_{i}:=u_{N}(z)\left(\mathbf{1}^{t} \Sigma_{2}^{-1}\right)_{i}=\frac{u_{N}(z)(g(0)-g(\alpha-\beta))}{g(0)^{2}-g(\alpha-\beta)^{2}}=\frac{u_{N}(z)}{g(0)+g(\alpha-\beta)}, \quad i=1,2 .
$$

Exploiting (3.2) we have

$$
\mathrm{P}\left(\varphi_{\alpha}>u_{N}(z), \varphi_{\beta}>u_{N}(z)\right) \leq \frac{1}{2 \pi} \frac{1}{\left|\operatorname{det} \Sigma_{2}\right|^{1 / 2} \Delta_{1} \Delta_{2}} \exp \left(-\frac{u_{N}(z)^{2}}{2} \mathbf{1}^{t} \Sigma_{2}^{-1} \mathbf{1}\right)
$$




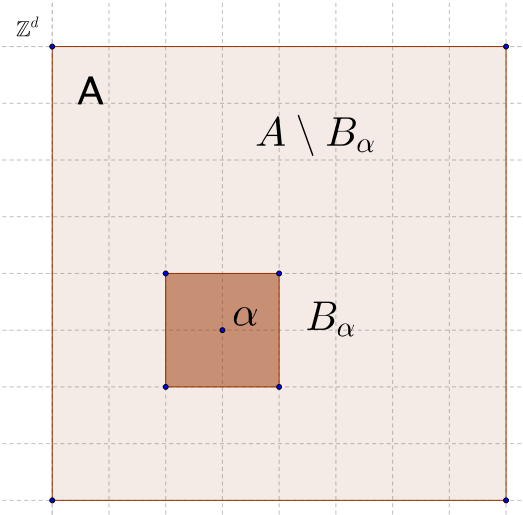

(A) $B_{\alpha}$ when $\alpha \in \operatorname{int}(A)$.

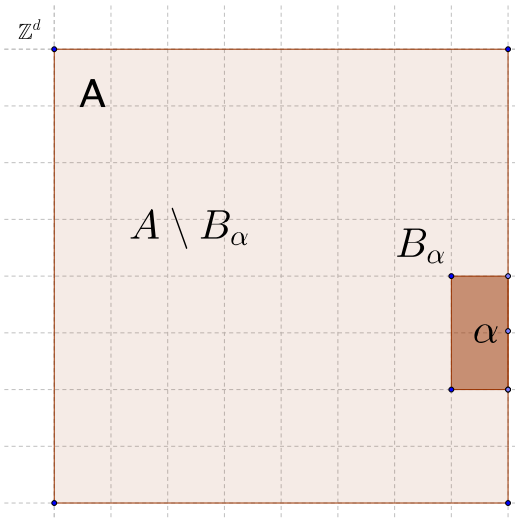

(в) $B_{\alpha}$ when $\alpha \in \partial A$.

Figure 3.1. Examples of $B_{\alpha}$

Note that using the explicit formula for the determinant one can bound the first factor easily by

$$
\frac{1}{2 \pi} \frac{1}{\left|\operatorname{det} \Sigma_{2}\right|^{1 / 2} \Delta_{1} \Delta_{2}} \leq \frac{\left(1+\frac{g(\alpha-\beta)}{g(0)}\right)^{3 / 2}}{\left(1-\frac{g(\alpha-\beta)}{g(0)}\right)^{1 / 2}} .
$$

Now using $u_{N}(z)^{2}=b_{N}^{2}+2 g(0) z+g(0)^{2} z^{2} / b_{N}^{2}$ and the bound of $b_{N}^{2}$ by Hall (1982, Equation 3)

$$
g(0)(2 \log N-\log \log N-\log 4 \pi) \leq b_{N}^{2} \leq 2 g(0) \log N
$$


we can now upper bound the exponential term by,

$$
\exp \left(-\frac{u_{N}(z)^{2}}{2} \mathbf{1}^{t} \Sigma_{2}^{-1} \mathbf{1}\right) \leq N^{-\frac{2 g(0)}{g(0)+g(\alpha-\beta)}} \mathrm{e}^{-\frac{2 g(0) z}{g(0)+g(\alpha-\beta)}+\mathrm{o}(1)} .
$$

Also note that for $x \neq 0, g(\|x\|) / g(0) \leq g\left(e_{1}\right) / g(0)=1-\kappa$ where $\kappa:=$ $\mathbb{P}_{0}\left(\widetilde{H}_{0}=+\infty\right) \in(0,1)$ and $\widetilde{H}_{0}=\inf \left\{n \geq 1: S_{n}=0\right\}$. Hence we have that

$$
\frac{g(0)}{g(0)+g(\alpha-\beta)} \geq \frac{1}{2-\kappa} \quad \text { and } \quad \frac{g(\alpha-\beta)}{g(0)+g(\alpha-\beta)} \leq 1-\kappa .
$$

We thus obtain

$$
\begin{aligned}
& \mathrm{P}\left(\varphi_{\alpha}>u_{N}(z), \varphi_{\beta}>u_{N}(z)\right) \\
& \leq \frac{(2-\kappa)^{3 / 2}}{\kappa^{1 / 2}} N^{-\frac{2}{(2-\kappa)}} \max \left(\mathrm{e}^{-2 z} \mathbb{1}_{\{z \leq 0\}}, \mathrm{e}^{-2 z /(2-\kappa)} \mathbb{1}_{\{z>0\}}\right) .
\end{aligned}
$$

We get finally for some constants $c, c^{\prime}>0$ depending only on $d$ and $\kappa$

$$
\begin{aligned}
b_{2} & \leq c N(\log N)^{d(2+2 \epsilon)} \frac{(2-\kappa)^{3 / 2}}{\kappa^{1 / 2}} N^{-\frac{2}{(2-\kappa)}} \max \left(\mathrm{e}^{-2 z} \mathbb{1}_{\{z \leq 0\}}, \mathrm{e}^{-2 z /(2-\kappa)} \mathbb{1}_{\{z>0\}}\right) \\
& \leq c^{\prime} N^{-\frac{\kappa}{(2-\kappa)}}(\log N)^{d(2+2 \epsilon)} \max \left(\mathrm{e}^{-2 z} \mathbb{1}_{\{z \leq 0\}}, \mathrm{e}^{-2 z /(2-\kappa)} \mathbb{1}_{\{z>0\}}\right) .
\end{aligned}
$$

Since $\kappa /(2-\kappa)>0$ we have that $b_{2}=o(1)$.

(iii) Recall $b_{3}=\sum_{\alpha \in A} \mathrm{E}\left[\left|\mathrm{E}\left[X_{\alpha}-p_{\alpha} \mid \mathcal{H}_{\alpha}\right]\right|\right]$. It will be convenient to introduce also another $\sigma$-algebra which strictly contains $\mathcal{H}_{\alpha}=\sigma\left(X_{\beta}: \beta \in A \backslash B_{\alpha}\right)$, that is

$$
\mathcal{H}_{\alpha}^{\prime}=\sigma\left(\varphi_{\beta}: \beta \in A \backslash B_{\alpha}\right) .
$$

Using the tower property of the conditional expectation and Jensen's inequality

$$
\mathrm{E}\left[\left|\mathrm{E}\left[X_{\alpha}-p \mid \mathcal{H}_{\alpha}\right]\right|\right] \leq \mathrm{E}\left[\left|\mathrm{E}\left[X_{\alpha}-p \mid \mathcal{H}_{\alpha}^{\prime}\right]\right|\right] .
$$

At this point we recognize, thanks to Corollary 2.1, that

$$
\mathrm{E}\left[X_{\alpha} \mid \mathcal{H}_{\alpha}^{\prime}\right]=\widetilde{\mathrm{P}}_{\mathbb{Z}^{d} \backslash\left(A \backslash B_{\alpha}\right)}\left(\psi_{\alpha}+\mu_{\alpha}>u_{N}(z)\right) \quad \mathrm{P}-a . s .
$$

where $\left(\psi_{\alpha}\right)_{\alpha \in \mathbb{Z}^{d}}$ is a Gaussian Free Field with zero boundary conditions outside $A \backslash B_{\alpha}$. In addition, setting $U_{\alpha}:=\mathbb{Z}^{d} \backslash\left(A \backslash B_{\alpha}\right)$, we observe that $g_{U_{\alpha}}(\alpha) \leq g(0)$ Lawler (1991, Section 1.5).

We will make use of the fact that $\mu_{\alpha}$ is a centered Gaussian, and apply the same estimates of Popov and Ráth (2015): first observe using strong Markov property we have $\beta \in A \backslash B_{\alpha}$,

$$
g(\alpha, \beta)=\sum_{\gamma \in A \backslash B_{\alpha}} \mathbb{P}_{\alpha}\left(H_{A \backslash B_{\alpha}}<+\infty, S_{H_{A \backslash B_{\alpha}}}=\gamma\right) g(\gamma, \beta) .
$$

We can plug this in to obtain

$$
\begin{aligned}
& \operatorname{Var}\left[\mu_{\alpha}\right] \stackrel{(3.6)}{=} \sum_{\beta \in A \backslash B_{\alpha}} \mathbb{P}_{\alpha}\left(H_{A \backslash B_{\alpha}}<+\infty, S_{H_{A \backslash B_{\alpha}}}=\beta\right) g(\alpha, \beta) \leq \sup _{\beta \in A \backslash B_{\alpha}} g(\alpha, \beta) \\
& \leq \frac{c}{(\log N)^{2(1+\epsilon)(d-2)}}
\end{aligned}
$$

by the standard estimates for the Green's function

$$
c_{d}\|\alpha-\beta\|^{2-d} \leq g(\alpha, \beta) \leq C_{d}\|\alpha-\beta\|^{2-d}
$$


for some $0<c_{d} \leq C_{d}<+\infty$ independent of $\alpha$ and $\beta$ Lawler (1991, Theorem 1. 5. 4). Using the estimate

$$
\mathrm{P}(|\mathcal{N}(0,1)|>a) \leq \mathrm{e}^{-a^{2} / 2}, a>0
$$

we get that there exists a constant $C>0$ such that

$$
\mathrm{P}\left(\left|\mu_{\alpha}\right|>\left(u_{N}(z)\right)^{-1-\epsilon}\right) \leq C \exp \left(-(\log N)^{(2 d-5)(1+\epsilon)}\right) .
$$

Note that this quantity goes to zero since $d \geq 3$. Hence

$$
\begin{aligned}
& \mathrm{E}\left[\left|\widetilde{\mathrm{P}}_{U_{\alpha}}\left(\psi_{\alpha}+\mu_{\alpha}>u_{N}(z)\right)-p\right|\right] \\
& =\mathrm{E}\left[\left|\widetilde{\mathrm{P}}_{U_{\alpha}}\left(\psi_{\alpha}+\mu_{\alpha}>u_{N}(z)\right)-p\right| \mathbb{1}_{\left\{\left|\mu_{\alpha}\right| \leq\left(u_{N}(z)\right)^{-1-\epsilon}\right\}}\right] \\
& +\mathrm{E}\left[\left|\widetilde{\mathrm{P}}_{U_{\alpha}}\left(\psi_{\alpha}+\mu_{\alpha}>u_{N}(z)\right)-p\right| \mathbb{1}_{\left\{\left|\mu_{\alpha}\right|>\left(u_{N}(z)\right)^{-1-\epsilon}\right\}}\right]=: T_{1}+T_{2} .
\end{aligned}
$$

By (3.10) and the fact that $d \geq 3$, we notice that $N T_{2}=o(1)$. Therefore it is sufficient to treat the term $T_{1}$. By conditioning on whether $p$ is larger or smaller than $\mathrm{P}_{U_{\alpha}}\left(\psi_{\alpha}+\mu_{\alpha}>u_{N}(z)\right)$ we can split the event in $T_{1}$ into the following two terms.

$$
\begin{aligned}
& \mathrm{E}\left[\left(\widetilde{\mathrm{P}}_{U_{\alpha}}\left(\psi_{\alpha}+\mu_{\alpha}>u_{N}(z)\right)-p\right) \mathbb{1}_{\left\{\left|\mu_{\alpha}\right| \leq\left(u_{N}(z)\right)^{-1-\epsilon}\right\}} \mathbb{1}_{\left\{p<\widetilde{\mathrm{P}}_{U_{\alpha}}\left(\psi_{\alpha}+\mu_{\alpha}>u_{N}(z)\right)\right\}}\right] \\
&+\mathrm{E}\left[\left(p-\widetilde{\mathrm{P}}_{U_{\alpha}}\left(\psi_{\alpha}+\mu_{\alpha}>u_{N}(z)\right)\right) \mathbb{1}_{\left\{\left|\mu_{\alpha}\right| \leq\left(u_{N}(z)\right)^{-1-\epsilon}\right\}} \mathbb{1}_{\left\{p \geq \widetilde{\mathrm{P}}_{U_{\alpha}}\left(\psi_{\alpha}+\mu_{\alpha}>u_{N}(z)\right)\right\}}\right] \\
&=: T_{1,1}+T_{1,2} .
\end{aligned}
$$

We will now deal with $T_{1,2}$. The first one can be treated with a similar calculation. Using Mills ratio and fact that $\psi_{\alpha}$ has variance $g_{U_{\alpha}}$ we get that,

$$
\begin{aligned}
& p-\widetilde{\mathrm{P}}_{U_{\alpha}}\left(\psi_{\alpha}+\mu_{\alpha}>u_{N}(z)\right) \\
& \leq \frac{\sqrt{g(0)} \mathrm{e}^{-\frac{u_{N}(z)^{2}}{2 g(0)}}}{\sqrt{2 \pi} u_{N}(z)}-\left(1-\left(\frac{\sqrt{g_{U_{\alpha}}(\alpha)}}{u_{N}(z)-\mu_{\alpha}}\right)^{2}\right) \frac{\sqrt{g_{U_{\alpha}}(\alpha)} \mathrm{e}^{-\frac{\left(u_{N}(z)-\mu_{\alpha}\right)^{2}}{2 g_{U_{\alpha}(\alpha)}}}}{\sqrt{2 \pi}\left(u_{N}(z)-\mu_{\alpha}\right)}
\end{aligned}
$$

We have on the event $\left\{\left|\mu_{\alpha}\right| \leq\left(u_{N}(z)\right)^{-1-\epsilon}\right\}$ that the above is bounded by

$$
\begin{aligned}
& \frac{\sqrt{g(0)} \mathrm{e}^{-\frac{u_{N}(z)^{2}}{2 g(0)}}}{\sqrt{2 \pi} u_{N}(z)} \times \\
& \times\left(1-(1+\mathrm{o}(1)) \frac{\sqrt{g_{U_{\alpha}}(\alpha)} u_{N}(z) \mathrm{e}^{\left(1-\frac{g(0)}{g_{U_{\alpha}}(\alpha)}\right) \frac{u_{N}(z)^{2}}{2 g(0)}+\frac{u_{N}(z)^{-\epsilon}}{g_{U_{\alpha}}(\alpha)}-\frac{u_{N}(z)^{-2-2 \epsilon}}{2 g_{U_{\alpha}}(\alpha)}}}{\sqrt{g(0)} u_{N}(z)\left(1-u_{N}(z)^{-2-\epsilon}\right)}\right) .
\end{aligned}
$$

Since the bound is non random, by bounding the indicator functions by 1 ,

$$
\mathrm{E}\left[\left(p-\widetilde{\mathrm{P}}_{U_{\alpha}}\left(\psi_{\alpha}+\mu_{\alpha}>u_{N}(z)\right)\right) \mathbb{1}_{\left\{\left|\mu_{\alpha}\right| \leq\left(u_{N}(z)\right)^{-1-\epsilon}\right\}} \mathbb{1}_{\left\{p \geq \widetilde{\mathrm{P}}_{U_{\alpha}}\left(\psi_{\alpha}+\mu_{\alpha} \leq u_{N}(z)\right)\right\}}\right]
$$

is bounded above by (3.13). Now

$$
b_{3} \leq \sum_{\alpha \in A}\left(T_{1}+T_{2}\right) \stackrel{(3.10)}{\leq} \sum_{\alpha \in A} T_{1}+\mathrm{o}(1)=\sum_{\alpha \in A} T_{1,1}+\sum_{\alpha \in A} T_{1,2}+\mathrm{o}(1) .
$$


Then

$$
\begin{aligned}
T_{1,2} & =\frac{\sqrt{g(0)} \mathrm{e}^{-\frac{u_{N}(z)^{2}}{2 g(0)}}}{\sqrt{2 \pi} u_{N}(z)} \times \\
& \times\left(1-(1+\mathrm{o}(1))\left(\frac{\sqrt{g_{U_{\alpha}}(\alpha)} u_{N}(z) \mathrm{e}^{\left(1-\frac{g(0)}{g_{\alpha}(\alpha)}\right) \frac{u_{N}(z)^{2}}{2 g(0)}+\mathrm{o}(1)}}{\sqrt{g(0)} u_{N}(z)(1+\mathrm{o}(1))}\right)\right) .
\end{aligned}
$$

Observe that $1-\frac{g(0)}{g_{U_{\alpha}}(\alpha)}<0$ since $g(0)>g_{U_{\alpha}}(\alpha)$. We observe further that (and we will prove it in a moment)

Claim 3.2. $\sup _{\alpha \in A}\left(1-\frac{g(0)}{g_{U_{\alpha}}(\alpha)}\right) u_{N}(z)^{2}=\mathrm{o}(1)$.

Therefore $T_{1,2}=\mathrm{o}(1)$ uniformly in $\alpha$. This yields that

$$
\sum_{\alpha \in A} T_{1,2} \leq N \frac{\sqrt{g(0)} \mathrm{e}^{-\frac{u_{N}(z)^{2}}{2 g(0)}}}{\sqrt{2 \pi} u_{N}(z)} \mathrm{o}(1)=\mathrm{e}^{-z+\mathrm{o}(1)} \mathrm{o}(1) .
$$

Analogously, $\sum_{\alpha \in A} T_{1,1}=\mathrm{o}(1)$. Plugging (3.15) in (3.14), one obtains $b_{3}=\mathrm{o}(1)$. We now only need to show Claim 3.2. By the Markov property we know

$$
g_{U_{\alpha}}(\alpha)=g(0)-\sum_{\gamma \in A \backslash B_{\alpha}} \mathbb{P}_{\alpha}\left(H_{A \backslash B_{\alpha}}<+\infty, S_{H_{A \backslash B_{\alpha}}}=\gamma\right) g(\gamma, \alpha) .
$$

This shows that

$$
0 \leq \frac{g(0)}{g_{U_{\alpha}}(\alpha)}-1 \leq \frac{\sup _{\gamma \in A \backslash B_{\alpha}} g(\gamma, \alpha)}{g_{U_{\alpha}}(\alpha)}
$$

Note that $g(\gamma, \alpha) \stackrel{(3.8)}{\leq} C_{d}(\log N)^{-2(d-2)(1+\epsilon)}$. Also,

$$
g_{U_{\alpha}}(\alpha)=\mathbb{E}_{\alpha}\left[\sum_{n=0}^{H_{A \backslash B_{\alpha}}} \mathbb{1}_{\left\{S_{n}=\alpha\right\}}\right] \geq 1
$$

and hence we have

$$
0 \leq \frac{g(0)}{g_{U_{\alpha}}(\alpha)}-1 \leq c(\log N)^{-2(d-2)(1+\epsilon)}
$$

from which it follows that

$$
\left(1-\frac{g(0)}{g_{U_{\alpha}}(\alpha)}\right) u_{N}(z)^{2} \leq c(\log N)^{-2(d-2)(1+\epsilon)}(\log N+z+\mathrm{o}(1))=\mathrm{o}(1) .
$$

Therefore the claim follows and we have shown pointwise convergence.

3.2. DGFF with boundary conditions: proof of Theorem 1.2. The idea of the proof is to exploit the convergence we have obtained in the previous section. We will show a lower bound through a comparison with i. i. d. variables, and an upper bound by considering the maximum restricted to the bulk of $V_{N}$, concluding by means of a convergence-of-types result. We abbreviate $g_{N}(\cdot, \cdot):=g_{V_{N}}(\cdot, \cdot)$. For $\delta>0$ define (recall that $V_{N}=[0, n-1]^{d} \cap \mathbb{Z}^{d}$, with $N=n^{d}$ )

$$
V_{N}^{\delta}:=\left\{\alpha \in V_{N}:\|\alpha-\gamma\|>\delta N^{1 / d}, \gamma \in \mathbb{Z}^{d} \backslash V_{N}\right\} .
$$

We begin with the easier lower bound. 
Proof of Theorem 1.2: lower bound: We will need a lower and an upper bound on the limiting distribution of the maximum. Let us start with the former. We use the shortcut $\widetilde{\mathrm{P}}_{N}:=\widetilde{\mathrm{P}}_{V_{N}}$. First we note that since the covariance of $\left(\psi_{\alpha}\right)$ is nonnegative, we can apply Slepian's lemma for the lower bound. Let $\left(Z_{\alpha}\right)_{\alpha \in V_{N}}$ be independent mean zero Gaussian variables with variance $g_{N}(\alpha)$; then by Slepian's lemma it follows that

$$
\widetilde{\mathrm{P}}_{N}\left(\max _{\alpha \in V_{N}} Z_{\alpha} \leq u_{N}(z)\right) \leq \widetilde{\mathrm{P}}_{N}\left(\max _{\alpha \in V_{N}} \psi_{\alpha} \leq u_{N}(z)\right),
$$

where $u_{N}(z)=a_{N} z+b_{N}$ as before. Then we want to analyze $\mathrm{P}\left(\max _{\alpha \in A} Z_{\alpha} \leq\right.$ $u_{N}(z)$ ). First fix $z \in \mathbb{R}$. Take $N$ large enough such that $-g(0) b_{N}^{2} \leq z$ (this is possible as $\left.b_{N}^{2} \rightarrow+\infty\right)$. Now note that

$$
\begin{aligned}
& \widetilde{\mathrm{P}}_{N}\left(\max _{\alpha \in V_{N}} Z_{\alpha} \leq u_{N}(z)\right)=\prod_{\alpha \in V_{N}}\left(1-\widetilde{\mathrm{P}}_{N}\left(Z_{\alpha}>u_{N}(z)\right)\right) \\
& \stackrel{(3.1)}{\geq} \prod_{\alpha \in V_{N}}\left(1-\frac{\mathrm{e}^{-\frac{u_{N}(z)^{2}}{2 g_{N}(\alpha)}}}{\sqrt{2 \pi} u_{N}(z)} \sqrt{g_{N}(\alpha)}\right) \geq\left(1-\frac{\mathrm{e}^{-\frac{u_{N}(z)^{2}}{2 g(0)}}}{\sqrt{2 \pi} u_{N}(z)} \sqrt{g(0)}\right)^{N} .
\end{aligned}
$$

The last term converges to $\exp \left(-\mathrm{e}^{-z}\right)$ as $N \rightarrow+\infty$. This shows that for any fixed $z \in \mathbb{R}$,

$$
\liminf _{N \rightarrow+\infty} \widetilde{\mathrm{P}}_{N}\left(\max _{\alpha \in V_{N}} \psi_{\alpha} \leq u_{N}(z)\right) \geq \exp \left(-\mathrm{e}^{-z}\right)
$$

In order to prove the upper bound of Theorem 1.2, we shall need a Lemma which will allow us to derive the convergence of the maximum in $V_{N}$ from that of the maximum in $V_{N}^{\delta}$.

Lemma 3.3. Let $N \geq 1, F_{N}$ be a distribution function, and $m_{N}=(1-2 \delta)^{d} N$. Let $a_{N}$ and $b_{N}$ be as in (1.1). If $\lim _{N \rightarrow+\infty} F_{N}\left(a_{m_{N}} z+b_{m_{N}}\right)=\exp \left(-\mathrm{e}^{-z}\right)$, then

$$
\lim _{N \rightarrow+\infty} F_{N}\left(a_{N} z+b_{N}\right)=\exp \left(-\mathrm{e}^{-z+d \log (1-2 \delta)}\right) .
$$

Proof: The proof follows from a convergence-of-types theorem (see Resnick, 1987, Proposition 0.2) if we can show that

$$
\frac{a_{m_{N}}}{a_{N}} \rightarrow 1 \quad \text { and } \quad \frac{b_{m_{N}}-b_{N}}{a_{N}} \rightarrow d \log (1-2 \delta) .
$$

It is easy to see that

$$
\frac{a_{m_{N}}}{a_{N}} \sim\left(1+\frac{d \log (1-2 \delta)}{\log N}\right)^{1 / 2} \rightarrow 1
$$

To show the second asymptotics note that

$$
\sqrt{2 g(0) \log m_{N}}-\sqrt{2 g(0) \log N}=\left[\frac{d \log (1-2 \delta)}{2 \log N}+\mathrm{O}\left(\frac{1}{(\log N)^{2}}\right)\right] \sqrt{2 g(0) \log N} .
$$


Also observe that as $N \rightarrow+\infty$ one gets

$$
\begin{aligned}
& \sqrt{g(0)}\left[\frac{\log \log (4 \pi N)}{2 \sqrt{2 \log N}}-\frac{\log \log \left(4 \pi m_{N}\right)}{2 \sqrt{2 \log m_{N}}}\right] \\
& =\frac{\sqrt{g(0)}}{2 \sqrt{2 \log N}}\left[-\log \left(1+\frac{d \log (1-2 \delta)}{\log N}\right)+o(1)\right] .
\end{aligned}
$$

(3.19) and (3.20) together give

$$
b_{m_{N}}-b_{N}=\sqrt{g(0)} \frac{d \log (1-2 \delta)}{\sqrt{2 \log N}}+\mathrm{O}\left((\log N)^{-\frac{3}{2}}\right) .
$$

Using $a_{N}^{-1}=g(0)^{-1} \sqrt{2 g(0) \log N}(1+\mathrm{o}(1))$ and the above we get that

$$
\frac{b_{m_{N}}-b_{N}}{a_{N}} \rightarrow d \log (1-2 \delta)
$$

We have now the tools to finish the upper bound.

Proof of Theorem 1.2: upper bound: First fix $z \in \mathbb{R}$ and $\delta>0$, set $m_{N}:=\left|V_{N}^{\delta}\right|=$ $(1-2 \delta)^{d} N$. For the upper bound, we again use Lemma 2.1 and the fact that, for $\alpha \in V_{N}^{\delta}$, one has the equality $\varphi_{\alpha}=\psi_{\alpha}+\mu_{\alpha}^{(N)}$ under the infinite volume measure $\mathrm{P}$, where $\mu_{\alpha}^{(N)}=\mathrm{E}\left[\varphi_{\alpha} \mid \mathcal{F}_{\partial V_{N}}\right]$. Hence if we fix $\varepsilon>0$, and condition on the event that

$$
\left\{\max _{\alpha \in V_{N}^{\delta}}\left|\mu_{\alpha}^{(N)}\right| \leq \varepsilon a_{m_{N}}\right\}
$$

(where $a_{m_{N}}$ is defined according to (1.1)) we have

$$
\begin{aligned}
\widetilde{\mathrm{P}}_{N}\left(\max _{\alpha \in V_{N}} \psi_{\alpha} \leq u_{m_{N}}(z)\right) & \leq \mathrm{P}\left(\max _{\alpha \in V_{N}^{\delta}} \varphi_{\alpha} \leq u_{m_{N}}(z+\epsilon)\right) \\
& +\mathrm{P}\left(\max _{\alpha \in V_{N}^{\delta}}\left|\mu_{\alpha}\right|>\varepsilon a_{m_{N}}\right) .
\end{aligned}
$$

First we show that the second term goes to zero. Observe that $\mu_{\alpha}$ is a centered Gaussian with variance

$$
\max _{\beta \in V_{N}^{\delta}} \operatorname{Var}\left[\mu_{\beta}\right] \leq \sup _{\beta \in V_{N}^{\delta}, \gamma \in \partial V_{N}} g(\beta, \gamma)=\mathrm{O}\left(N^{(2-d) / d}\right) .
$$

Let $\left(\Phi_{\alpha}\right)_{\alpha \in V_{N}^{\delta}}$ be a collection of i.i.d. Gaussians with mean zero and $\mathrm{E}\left[\Phi_{\alpha}^{2}\right]=$ $\mathrm{E}\left[\mu_{\alpha}^{2}\right]$ for all $\alpha$. By Slepian and Talagrand (2003, Prop. 1.1.3) we have

$$
\begin{aligned}
\mathrm{P}\left(\max _{\alpha \in V_{N}^{\delta}}\left|\mu_{\alpha}\right|>\varepsilon a_{m_{N}}\right) & \leq \frac{2 \mathrm{E}\left[\max _{\alpha \in V_{N}^{\delta}} \Phi_{\alpha}\right]}{a_{m_{N}} \varepsilon}+2 \mathrm{P}\left(\max _{\alpha \in V_{N}^{\delta}} \Phi_{\alpha} \leq 0\right) \\
& \leq \frac{2 \sqrt{\max _{\beta \in V_{N}^{\delta}} \operatorname{Var}\left[\mu_{\beta}\right] \log \left|V_{N}^{\delta}\right|}}{a_{m_{N}} \varepsilon}+\mathrm{o}(1) .
\end{aligned}
$$


Since $a_{N}$ grows like $(\sqrt{2 \log N})^{-1}$ as $N \rightarrow+\infty$, we can conclude that, for every $\varepsilon>0$

$$
\lim _{N \rightarrow+\infty} \mathrm{P}\left(\max _{\alpha \in V_{N}^{\delta}}\left|\mu_{\alpha}\right|>\varepsilon a_{m_{N}}\right)=0 .
$$

Using Theorem 1.1 we have that

$$
\lim _{N \rightarrow+\infty} \mathrm{P}\left(\max _{\alpha \in V_{N}^{\delta}} \varphi_{\alpha} \leq u_{m_{N}}(z+\epsilon)\right)=\exp \left(-\mathrm{e}^{-(z+\epsilon)}\right) .
$$

Using continuity of the limit above and letting $\varepsilon \rightarrow 0$ in the above we obtain from (3.21),

$$
\limsup _{N \rightarrow+\infty} \widetilde{\mathrm{P}}_{N}\left(\max _{\alpha \in V_{N}^{\delta}} \psi_{\alpha} \leq u_{m_{N}}(z)\right) \leq \exp \left(-\mathrm{e}^{-z}\right) .
$$

Now using an easy comparison with independent random variables just as in the proof of the lower bound of Theorem 1.2 above it follows that (3.22) is in fact an equality. By Lemma 3.3 one can conclude that

$$
\lim _{N \rightarrow+\infty} \widetilde{\mathrm{P}}_{N}\left(\max _{\alpha \in V_{N}^{\delta}} \psi_{\alpha} \leq u_{N}(z)\right)=\exp \left(-\mathrm{e}^{-z+d \log (1-2 \delta)}\right)
$$

and thus letting $\delta \rightarrow 0$, the upper bound follows.

\section{Acknowledgements}

The authors would like to thank an anonymous referee for several useful comments and remarks.

\section{References}

R. J. Adler and J. E. Taylor. Random fields and geometry. Springer Monographs in Mathematics. Springer, New York (2007). ISBN 978-0-387-48112-8. MR2319516.

R. Arratia, L. Goldstein and L. Gordon. Two moments suffice for Poisson approximations: the Chen-Stein method. Ann. Probab. 17 (1), 9-25 (1989). MR972770.

M. Biskup and O. Louidor. Conformal symmetries in the extremal process of two-dimensional discrete gaussian free field. ArXiv Mathematics e-prints (2014). arXiv: 1410.4676.

M. Biskup and O. Louidor. Extreme Local Extrema of Two-Dimensional Discrete Gaussian Free Field. Comm. Math. Phys. 345 (1), 271-304 (2016). MR3509015. M. Bramson, J. Ding and O. Zeitouni. Convergence in law of the maximum of the two-dimensional discrete Gaussian free field. Comm. Pure Appl. Math. 69 (1), 62-123 (2016). MR3433630.

S. Chatterjee. Superconcentration and related topics. Springer Monographs in Mathematics. Springer, Cham (2014). ISBN 978-3-319-03885-8. MR3157205.

A. Drewitz and P.-F. Rodriguez. High-dimensional asymptotics for percolation of Gaussian free field level sets. Electron. J. Probab. 20, no. 47, 39 (2015). MR3339867. 
T. Funaki. Stochastic interface models. In Lectures on probability theory and statistics, volume 1869 of Lecture Notes in Math., pages 103-274. Springer, Berlin (2005). MR2228384.

H.-O. Georgii. Gibbs measures and phase transitions, volume 9 of de Gruyter Studies in Mathematics. Walter de Gruyter \& Co., Berlin (1988). ISBN 0-89925-462-4. MR956646.

P. Hall. On the rate of convergence in the weak law of large numbers. Ann. Probab. 10 (2), 374-381 (1982). MR647510.

G. F. Lawler. Intersections of random walks. Probability and its Applications. Birkhäuser Boston, Inc., Boston, MA (1991). ISBN 0-8176-3557-2. MR1117680.

S. Popov and B. Ráth. On decoupling inequalities and percolation of excursion sets of the Gaussian free field. J. Stat. Phys. 159 (2), 312-320 (2015). MR3325312.

S. I. Resnick. Extreme values, regular variation, and point processes, volume 4 of Applied Probability. A Series of the Applied Probability Trust. Springer-Verlag, New York (1987). ISBN 0-387-96481-9. MR900810.

P.-F. Rodriguez and A.-S. Sznitman. Phase transition and level-set percolation for the Gaussian free field. Comm. Math. Phys. 320 (2), 571-601 (2013). MR3053773.

I. R. Savage. Mill's ratio for multivariate normal distributions. J. Res. Natl. Bur. Stand. Sec. B: Math.83 Math. Phys. 66B (3), 93-96 (1962). MR3053773.

A.-S. Sznitman. A lower bound on the critical parameter of interlacement percolation in high dimension. Probab. Theory Related Fields 150 (3-4), 575-611 (2011). MR2824867.

A.-S. Sznitman. Topics in occupation times and Gaussian free fields. Zurich Lectures in Advanced Mathematics. European Mathematical Society (EMS), Zürich (2012). ISBN 978-3-03719-109-5. MR2932978.

M. Talagrand. Spin glasses: a challenge for mathematicians, volume 46 of A Series of Modern Surveys in Mathematics. Springer-Verlag, Berlin (2003). ISBN 3-54000356-8. MR1993891. 